\title{
Épidémiologie et devenir des patients brûlés au Centre hospitalier universitaire Sylvanus Olympio de Lomé
}

\author{
Pikabalo Tchetikè, MD D - Tabana Mouzou, MD • Pilakimwé Egbohou, MD • \\ Gnimdou Akala-Yoba, MD • Lonlongnon Kossi Randolph, MD • Sarakawabalo Assenouwe, MD • \\ Alphonse Sonagnon Koudjo, MSc • Pitchaki Hemou, MD • Kadjika Tomta, MD
}

Received: 24 September 2019/Revised: 9 November 2019/ Accepted: 12 November 2019/Published online: 21 November 2019

(C) Canadian Anesthesiologists' Society 2019

La prise en charge des brûlés dans les pays à revenu faible comme le Togo se heurte à une inexistence de prise en charge préhospitalière, une rareté de centres de prise en charge spécialisée (CPS), et de personnels qualifiés. La mortalité de ce fait est plus élevée qu'ailleurs. ${ }^{1}$

Au Centre hospitalier universitaire Sylvanus Olympio (CHUSO) de Lomé, centre national de référence, les brûlés sont accueillis aux urgences chirurgicales (UC) où les premiers soins sont réalisés avant leur transfert selon la gravité jugée soit en service des brûlés ou en unité de réanimation polyvalente en l'absence de réanimation spécifiquement dédiée à la brûlure au sein d'un service naissant de brûlés.

Après approbation du comité d'éthique du CHUSO, nous avons réalisé une étude transversale, prospective sur une période de six mois du 20 avril au 20 octobre 2018, incluant tous les patients brûlés admis dans ce centre. Les données recueillies comprenaient: l'âge, le sexe, la profession, l'agent causal, le délai avant l'admission, la surface brûlée (SB), la profondeur, la prise en charge préhospitalière, l'utilisation d'un protocole de remplissage aux UC, la mise sous ventilation mécanique (VM), la prise en charge chirurgicale, et le devenir des patients. L'analyse des données était réalisée grâce au logiciel Statistical Package for Social Science, SPSS (version 21; IBM Corp., Armonk, NY, USA).

P. Tchetikè, MD $(\bowtie) \cdot$ T. Mouzou, MD · P. Egbohou, MD ·

G. Akala-Yoba, MD - L. K. Randolph, MD .

S. Assenouwe, MD - A. S. Koudjo, MSc - K. Tomta, MD

Service d'anesthésie réanimation CHU Sylvanus Olympio

(CHU SO), Lomé, Togo

e-mail: ftchet5@hotmail.fr

P. Hemou, MD

Service d'anesthésie réanimation Cochin, Paris, France
Un total de 85 patients brûlés sur $6159(1,4 \%)$ était admis aux UC durant la période d'étude. Aucun patient n'a eu de prise en charge préhospitalière. On notait une prédominance masculine $(62,2 \%)$, infantile $(58,8 \%)$ et de brûlure à l'eau chaude (50,6\%). Aucun patient n'a eu de chirurgie à la phase précoce ou aiguë.

On a enregistré neuf $(10,6 \%)$ décès. Les facteurs associés au décès sont répertoriés dans le tableau. On a enregistré plus de décès pour les $\mathrm{SB}>25 \%(30 \%)$ que celles $<25 \%(3,3 \%)$, rapport de cotes $(\mathrm{RC})=12,9$; IC (intervalle de confiance) $95 \%: 2,4$ à $68,3, P=0,001$. De même les brûlures du $2^{\text {ième }}$ degré profond, du $3^{\text {ième }}$ degré et en mosaïque étaient associées a plus de décès : $42,1 \%$ vs $1,5 \%$ pour celles du $1^{\text {er }}$ et $2^{\text {ième }}$ degré superficiel, RC 47,3 ; IC $95 \%: 5,4$ à $416, P=0,001$. Le recours à la VM $(66,7$ $\%)$, l'absence de protocole de remplissage $(4 \%)$ et la présence de lésions d'inhalation (50\%) étaient également associés au décès $(P<0,05)$. Par contre, l'âge, le délai avant l'admission, et le type de brûlure n'avais pas influencé le décès.

La fréquence des brûlures de 1,4\% dans cette étude est faible comparée aux données de la littérature, s'expliquant par l'absence de CPS, et l'orientation de ce fait des patients vers des hôpitaux périphériques non spécialisés, ou vers des tradithérapeutes ${ }^{2,3}$ La mortalité dans cette étude était de $10,6 \%$; celle rapportée dans la littérature varie de 5-55 $\%{ }^{4}$ Les facteurs prédictifs de mortalité retrouvés dans cette étude $\left(\mathrm{SB}>25 \%\right.$, les profondeurs $>$ au $2^{\text {ième }}$ degré superficiel, la VM, l'absence de protocole de remplissage, les lésions d'inhalation) étaient antérieurement rapportés. ${ }^{2,3,5}$ Parmi eux, le remplissage précoce reconnu comme déterminant majeur du pronostic du brûlé, reste un facteur corrigeable par une meilleure organisation des services préhospitaliers et la mise en place de protocoles de remplissage. ${ }^{5}$ L'association de la VM au mauvais devenir 
Tableau Facteurs associés aux décès par brûlure

\begin{tabular}{|c|c|c|c|c|c|}
\hline Facteurs & $\mathrm{N}^{1}$ & $\mathrm{~N}^{2}(\%)$ & $\mathrm{RC}$ & IC $95 \%$ & $P$ \\
\hline \multicolumn{6}{|l|}{ Age } \\
\hline$<15$ ans & 50 & $5(10,0)$ & 0,86 & 0,2 à 3,5 & 0,551 \\
\hline$>15$ ans & 35 & $4(11,3)$ & & & \\
\hline \multicolumn{6}{|l|}{ Délai d'admission } \\
\hline$<8 \mathrm{~h}$ & 57 & $7(11,8)$ & 1,6 & 0,3 à 8,4 & 0,440 \\
\hline$>8 \mathrm{~h}$ & 26 & $2(7,6)$ & & & \\
\hline \multicolumn{6}{|l|}{ Type de brûlure } \\
\hline Thermique & 78 & $9(11.5)$ & 1,1 & 1,0 à 1,2 & 0,443 \\
\hline Autres* & 7 & 0 & & & \\
\hline \multicolumn{6}{|l|}{ SB } \\
\hline$<25 \%$ & 61 & $2(3,3)$ & & & \\
\hline$>25 \%$ & 23 & $7(30,4)$ & 12,9 & 2,4 à 68,3 & 0,001 \\
\hline \multicolumn{6}{|l|}{ Profondeur } \\
\hline $1^{\mathrm{er}}+2^{\text {ième }}$ degré superficiel & 66 & $1(1,5)$ & & & \\
\hline $\begin{array}{l}2^{\text {ième }} \text { profond }+3^{\text {ième }} \\
\text { degré }+ \text { mosaïque }\end{array}$ & 19 & $8(42,1)$ & 47,3 & 5,4 à 416,0 & 0,001 \\
\hline \multicolumn{6}{|l|}{ Ventilation mécanique } \\
\hline Oui & 3 & $2(66,7)$ & 21,4 & 1,7 à 2,7 & 0,029 \\
\hline Non & 82 & $7(8,5)$ & & & \\
\hline \multicolumn{6}{|l|}{ Protocole de remplissage } \\
\hline Oui & 10 & $6(0,6)$ & 36 & & \\
\hline Non & 75 & $3(4,0)$ & 36 & 6,5 à 199,6 & 0,001 \\
\hline \multicolumn{6}{|l|}{ Lésion d'inhalation } \\
\hline Présence & 4 & $2(50)$ & 10,57 & 1,3 à 84,0 & 0,054 \\
\hline Absence & 81 & $7(8,6)$ & & & \\
\hline
\end{tabular}

Autres* = brûlures chimiques et électriques; $\mathrm{IC}=$ intervalle de confiance; $\mathrm{N}^{1}=$ effectif total; $\mathrm{N}^{2}=$ décès; $\mathrm{RC}=$ rapport de cotes; $\mathrm{SB}=$ surface brûlée

dans l'étude témoigne des efforts à faire dans la prise en charge en réanimation des brûlés.

L'absence de chirurgie à la phase précoce et aiguë dans cette étude s'explique par la non-disponibilité au Togo de chirurgien plasticien, reléguant cette prise en charge aux chirurgiens généralistes et traumatologues.

L'amélioration du devenir du patient brûlé au Togo passerait par une meilleure organisation des services d'urgences, la mise en place de CPS des brûlures et la formation de brûlologues.

\section{Conflit d'intérêt Aucun.}

\section{Déclaration de financement Aucun.}

Responsabilit' 'ditoriale Cet article a été traité par Dr Étienne de Médicis, rédacteur de la langue française, Journal canadien d'anesthésie.

\section{Références}

1. Le Dantec P, Niang B, Boulesteix G, Bellefleur JP, Pochan Y, Diatta $B$. Prise en charge de la brûlure, en milieu non spécialisé, en Afrique. Med Trop (Mars) 2003; 63: 567-72.

2. Edmond $K K$, Abhé $C M$, Yapo $Y P$, et al. Prise en charge des brûlures graves à Abidjan (Côte d'Ivoire). RAMUR 2013. Disponible à l'adresse URL: https://saranf.net/Prise-en-chargedes-brulures-156.html (consulté novembre 2019).

3. Ryan CM, Schoenfeld DA, Thorpe WP, Sheridan RL, Cassem EH, Tompkins $R G$. Objective estimates of the probability of death from burn injuries. N Engl J Med 1998; 338: 362-6.

4. Perro G, Bourdarias B, Cutillas M, Castède JC, Sanchez R. Analyse épidémiologique de 2000 brûlés hospitalisés à Bordeaux entre 1987 et 1994. Ann Burns Fire Disas 1996; vol. IX- n.3. Disponible à l'adresse URL: http://www.medbc.com/annals/ review/vol_9/num_3/text/vol9n3p131.htm (consulté novembre 2019).

5. Barrow RE, Jeschke $M G$, Herndon DN. Early fluid resuscitation improves outcomes in severely burned children. Resuscitation 2000; 45: 91-6.

Publisher's Note Springer Nature remains neutral with regard to jurisdictional claims in published maps and institutional affiliations. 